\title{
An enhanced apoptosis and a reduced angiogenesis are associated with the inhibition of lung colonisation in animals fed an $n-3$ polyunsaturated fatty acid-rich diet injected with a highly metastatic murine melanoma line
}

\author{
Antonella Mannini, Nadja Kerstin, Lido Calorini, Gabriele Mugnai and Salvatore Ruggieri* \\ Department of Experimental Pathology and Oncology, University of Florence, Viale G.B. Morgagni 50, 50134 Florence, Italy
}

(Received 7 January 2008 - Revised 10 April 2008 - Accepted 7 May 2008 - First published online 4 August 2008)

Both epidemiological and experimental studies indicate that dietary $n-3$ PUFA inhibit carcinogenesis and tumour growth. Metastatic diffusion has also been found to be affected in animals fed diets containing purified $n-3$ PUFA or fish oil. In the present study, we investigated whether the metastatic diffusion of a highly metastatic variant (F10-SR cells) isolated from the B16 melanoma F10 line was affected by feeding host animals a diet containing $5 \%$ fish oil. In these animals, compared with those fed a diet containing $5 \%$ maize oil, there was a reduced number of metastatic pulmonary colonies. The immunohistochemical analysis of appropriate markers revealed that the antimetastatic effect of dietary $n$-3 PUFA was not related to a reduction of proliferation, but rather to an enhanced apoptotic activity. The reduction of von Willebrand factor immunoreactivity found in pulmonary colonies of F10-SR cells grown in fish oil-fed animals indicates that a decrease of angiogenesis contributes to the antimetastatic effect of dietary n-3 PUFA. This conclusion stands in spite of the higher expression of vascular endothelial growth factor observed in pulmonary colonies grown in fish oil-fed animals.

B16 melanoma line: Fish oil diets: Metastases: Apoptosis: Angiogenesis

Although epidemiological data support a strong positive correlation between certain tumours and high-fat diets ${ }^{(1,2)}$, it is still debated how a high consumption of dietary lipids affects carcinogenesis. Animal studies provide evidence that a high content of linoleic acid $(18: 2 n-6)$ in the diet influences the development of carcinomas of the breast, colon and prostate, by acting at the promotional stage of carcinogenesis (for a review, see Carroll \& Khor $\left.^{(3)}\right)$. The reproduction of experimental metastasis has also been shown to be favoured by 18:2n-6-rich diets ${ }^{(4-7)}$. These experimental data prompted interest in $18: 2 n-6$ as a possible factor promoting the development of human tumours, in view of the fact that $18: 2 n-6$ is the most abundant fatty acid of the $n-6$ series in vegetable oils which are the major lipid component in the diets of the Western world. The promoting activity of $18: 2 n-6$ on carcinogenesis and tumour progression appears to be mediated by the bioactive metabolites of arachidonic acid (AA), the product of elongation and desaturation of $18: 2 n-6^{(8,9)}$.

More recently, epidemiological studies have reported that the risk of breast ${ }^{(10)}$, colon ${ }^{(11)}$ and prostate ${ }^{(12)}$ cancers is lower in populations whose dietary habits are characterised by a high intake of fish oil, which is particularly rich in EPA and DHA, the higher homologues of the $\alpha$-linolenic acid $(18: 3 n-3)$ family ${ }^{(13)}$. The protective effect of $n-3$ PUFA may also be derived from the observation of an enhancement of breast cancer rate ${ }^{(14)}$ among Japanese women during the past decades associated with a replacement of fish oil with maize oil in their diet. The recognition of an inhibitory activity of $n$-3 PUFA on tumour development was further supported by animal studies showing that feeding diets enriched with $n$-3 PUFA inhibited spontaneous and chemically induced carcinogenesis ${ }^{(15-17)}$, growth of mammary $^{(18-20)}$, colon $^{(21)}$ and liver carcinomas ${ }^{(22)}$ in rats and mice, and growth of human lung carcinoma in nude mice ${ }^{(23)}$.

Metastatic diffusion of melanoma ${ }^{(24)}$, colon ${ }^{(25)}$, mammary $^{(26)}$ and Lewis lung ${ }^{(27)}$ carcinomas was also inhibited when they were transplanted into animals fed n-3 PUFArich diets. In these studies, however, a few histotypes of malignant cells have been considered, and the effects of $n-3$ PUFA on metastatic diffusion have been examined by using various experimental approaches, for example, by feeding diets containing fish oil ${ }^{(26)}$ or purified EPA and DHA in variable proportions ${ }^{(25)}$, and by subcutaneous administration of fish oil ${ }^{(28)}$. Moreover, there are also a few studies that demonstrated a promoting activity of dietary $n-3$ PUFA on tumour growth and experimental metastasis ${ }^{(28,29)}$. Thus, more information should be available before introducing the use of appropriate dietary formulations for the chemoprevention and chemosuppression of human tumours.

To expand our knowledge about the inhibitory effects of dietary $n-3$ PUFA on metastatic diffusion, in the present study we investigated to what extent a diet containing $5 \%$ fish oil, compared with a diet containing $5 \%$ maize oil, affects the reproduction of experimental metastases developed from F10-SR 
cells, a highly metastatic variant isolated from the murine B16 melanoma F10 line. In the present study, we also investigated whether an alteration of the metastatic potential of F10-SR cells induced by our dietary protocol involves some biological activities crucial in tumour progression, such as cellular proliferation $^{(30)}$, apoptosis ${ }^{(31,32)}$ and angiogenesis ${ }^{(31,33)}$. Cellular proliferation, apoptosis and angiogenesis were examined by immunolocalisation of their respective markers in metastatic pulmonary colonies developed from F10-SR cells transplanted into animals fed diets containing maize oil or fish oil. These markers were: proliferating cell nuclear antigen $(\mathrm{PCNA})^{(34)}$, bax-bcl2 $2^{(35)}$, vascular endothelial growth factor $(\mathrm{VEGF})^{(36)}$ and endothelial-associated von Willebrand factor (vWF ${ }^{(37)}$.

\section{Materials and methods}

\section{Cells and culture conditions}

Cells used in the present study were a highly metastatic variant (F10-SR cells) isolated in our laboratory, from a metastasis reproduced by the murine B16 melanoma F10 line in adrenal gland. F10-SR cells $\left(5 \times 10^{5}\right)$ were seeded in $100 \mathrm{~mm}$ tissue culture dishes (Sarstedt), and grown in Dulbecco's modified Eagle's medium containing glucose $(4500 \mathrm{mg} / \mathrm{l})$ supplemented with $10 \%$ fetal calf serum (Gibco) at $37^{\circ} \mathrm{C}$ in a $10 \% \quad \mathrm{CO}_{2}$ humidified atmosphere. Cell cultures were propagated every $3 \mathrm{~d}$ by trypsinisation.

\section{Diets}

Diets used in the present study were: (a) a diet composed of $70 \%$ sucrose, $16 \%$ casein, $3 \% \alpha$-cellulose, $1 \%$ choline chloride, $1 \%$ vitamins, $4 \%$ salt mixture and $5 \%$ maize oil (maize oil diet); (b) an isoenergetic diet in which $5 \%$ maize oil was replaced with $5 \%$ fish oil (fish oil diet). The major fatty acids of the maize oil diet were: $14: 0,5.3 \% ; 16: 0,1.6 \%$; $16: 1,16.7 \% ; 18: 0,3.3 \% ; 18: 1,22.1 \% ; 18: 2,50.0 \%$. The fish oil diet contained: $14: 0,21.1 \% ; 16: 0,31.6 \% ; 16: 1$, $10.5 \% ; 18: 0,5.0 \% ; 18: 1,18.6 \% ; 18: 2,3.0 \% ; 20: 5 n-3$, $5.5 \% ; 22: 6 n-3,4.5 \%$. The maize oil and fish oil diets, supplied by Laboratorio Dottori Piccioni (Milan, Italy), were stored at $4^{\circ} \mathrm{C}$ in sealed plastic bags in vacuo.

\section{Animals and dietary treatments}

Newly weaned female mice (C57Bl/6 strain; Charles River, Frederick, MD, USA) were placed on the maize oil diet for 1 week, and then divided in two groups, each of six animals: one group continued to be fed the maize oil diet, while the other group was switched to the fish oil diet. The degree of $n$-3 PUFA incorporation into the liver phosphatidylcholine was experimentally evaluated in one representative animal by weekly measuring the EPA + DHA:AA ratio. The highest value of this ratio was reached in animals fed the fish oil diet after 5 weeks. At this moment, EPA + DHA:AA ratios in animals fed the maize oil or fish oil diet were $0 \cdot 2$ and $3 \cdot 7$, respectively. The food consumption (4.0 (SD 1.7) g/d per animal) and body weight of the experimental animals were assessed twice per week from the beginning of the dietary treatment to the end of the experiment. At the end of the experiments, animals showed a similar increase $(30.4 \%)$ of body weight, regardless of whether they were fed the maize oil or fish oil diet (data not shown).

Determination of lung colonisation of F10-SR cells
transplanted in animals fed maize oil or fish oil diets

A F10-SR cell suspension $\left(0 \cdot 2 \mathrm{ml} ; 5 \times 10^{5}\right.$ cells/ml Dulbecco's modified Eagle's medium) was injected into the lateral tail vein of experimental animals of the two groups fed their respective diets for 5 weeks. The animals were maintained at their respective diets until the end of experiment, i.e. 3 weeks after tumour cell injection. Animals were killed by cervical translocation under diethyl ether anaesthesia. Lung surface was inspected with the aid of a dissection microscope, and surface pulmonary colonies were counted, exsected from lung parenchyma and submitted to immunohistochemical analyses.

\section{Gas-chromatographic analyses of fatty acids}

Total lipids were extracted by the method of Folch et al. ${ }^{(38)}$ from liver homogenates. Phosphatidylcholine was separated from the other lipid classes by HPLC following the conditions reported in a previous paper ${ }^{(7)}$. Phosphatidylcholine, solubilised in $0.2 \mathrm{ml}$ benzene, was submitted to transmethylation with $5 \% \quad \mathrm{H}_{2} \mathrm{SO}_{4}$ in methanol, at $80^{\circ} \mathrm{C}$, overnight. The methyl-ester derivatives of fatty acids were analysed on a $30 \mathrm{~m} \times 0.53 \mathrm{~mm}$ internal diameter wide-bore capillary column (Megabore W DB-23, $50 \%$ cyanopropyl polysiloxan; J \& W Scientific, Folsom, CA, USA), mounted in a PerkinElmer gas chromatograph equipped with a hydrogen flame detector (PerkinElmer, Waltham, MA, USA). Fatty acid composition was determined from the total integrated area in the chromatogram. Percentages of EPA, DHA and AA were used to calculate EPA + DHA:AA ratios in liver phosphatidylcholine.

\section{Immunohistochemistry}

Immunoreactivities of PCNA, bax/bcl-2, VEGF and vWF were determined in $5 \mu \mathrm{m}$ thick sections of formalin-fixed paraffinembedded metastatic colonies, according to a procedure described in a previous paper ${ }^{(7)}$. Monoclonal antibodies against PCNA, bax/bcl-2 and VEGF were supplied by Santa Cruz Biotechnology (Santa Cruz, CA, USA), while the polyclonal antibody against mouse vWF was supplied by Dako (Glostrup, Denmark). Negative controls were represented by consecutive tissue sections submitted to the immunohistochemical procedure in which the primary antibodies were omitted.

The immunoreactivities of PCNA, bax, bcl-2 and VEGF were scored following a semi-quantitative procedure that graded the percentages of reactive cells as follows: + , focal (less than $10 \%) ;++$, moderate $(10$ to $50 \%) ;+++$, strong staining $(>50 \%$ stained cells $) ;-$, absent. Microvessel densities in the pulmonary colonies grown in animals fed the maize oil or fish oil diets were derived from the number of vWF-positive cells/microscopic field.

\section{Detection of apoptotic nuclei}

In order to confirm apoptotic activity in the pulmonary colonies developed in animals fed the maize oil or fish oil diets, DNA fragments in apoptotic nuclei were visualised according 
to the method of Gavrieli et al. ${ }^{(39)}$. This method is based on the reaction, catalysed by terminal deoxynucleotidyl transferase, of $3^{\prime}-\mathrm{OH}$ ends of DNA fragments with biotin-labelled deoxynucleotides (Oncogene Research Products, Cambridge, MA, USA). The biotinylated nucleotides were complexed with a streptavidin-horseradish peroxidase conjugate, followed by visualisation with diaminobenzidine chromogen. Sections were counterstained with a $0.3 \%$ methyl green solution and mounted on slides in a xylene mounting medium.

\section{Statistical analysis}

The Mann-Whitney test was used to determine the statistical significance of the differences between animals fed the maize oil diet and animals fed the fish oil diet in lung colonisation and microvessel densities.

\section{Results}

As shown in Fig. 1, F10-SR cells reproduced a lower number of metastatic colonies in the lungs of mice fed the fish oil diet, as compared with mice fed the maize oil diet. Regardless of the dietary regimen, PCNA (Figs. 2 (a) and (b)) was highly expressed in the pulmonary colonies developed in animals transplanted with F10-SR cells. Bax was moderately immunoreactive in the pulmonary colonies grown in animals fed the maize oil diet (Fig. 2 (c)), but highly expressed in the pulmonary colonies grown in animals fed the fish oil diet (Fig. 2 (d)). Regardless of the dietary regimen, pulmonary colonies did not express any reactivity for bcl-2 (see insert). Apoptotic nuclei, visualised by specific labelling of DNA fragments, were detectable only in metastatic colonies grown in animals fed the fish oil diet, as indicated by arrows in Fig. 2 (f).

As shown in Fig. 3, VEGF immunoreactivity was rather weak in the pulmonary colonies developed in animals fed the maize oil diet (Fig. 3 (a)), but fairly strong in colonies grown in animals fed the fish oil diet (Fig. 3 (b)). In these latter, however, there was a reduction of microvessel density, as determined by the visualisation of endothelial-associated vWF (Fig. 3 (d)).

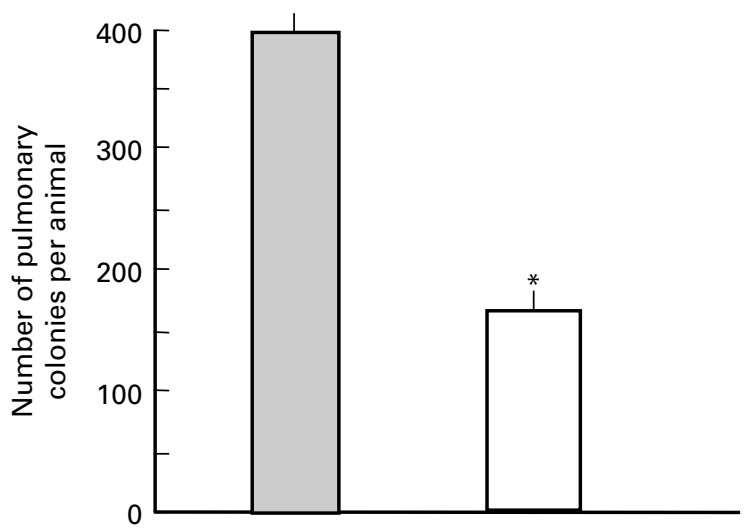

Fig. 1. Lung colonisation of F10-SR cells in animals fed maize oil $(\square)$ or fish oil $(\square)$ diets. Values are the means of number of lung colonies per animal ( $n 5)$, with standard errors represented by vertical bars. * Mean value was significantly different from that of the animals fed the maize oil diet $(P=0.004)$.

\section{Discussion}

In the present study, we demonstrated that the development of experimental metastases from a highly metastatic murine melanoma line, the F10-SR cells, was inhibited in animals fed a diet containing $5 \%$ fish oil, a concentration much lower than that used in other studies ${ }^{(40)}$. In contrast with the present results, Salem et al. ${ }^{(28)}$ found an accentuated growth of primary tumour and an increased development of lung metastases from B16 melanoma cells in mice treated with subcutaneous administration of fish oil before and after subcutaneous transplantation of tumour cells. The discrepancy between our and Salem's results might be related to the different experimental protocols used in the respective studies. An antimetastatic activity of $n$-3 PUFA was also found by Reich et al. ${ }^{(24)}$ who obtained a reduction of lung colonisation of B16-F10 melanoma cells which had been previously enriched in vitro with EPA, an observation also made in our laboratory (A Mannini and S Ruggieri, unpublished results). Moreover, Iigo et al. ${ }^{(25)}$ found an inhibition of lung colonisation in animals injected with colon carcinoma cells which had been previously submitted to a serial transplantation into mice fed an $n$-3 PUFArich diet. Reich's and Iigo's observations would suggest that the inhibitory activity of dietary $n-3$ PUFA on metastatic growth is mediated by their incorporation into tumour cells, although a contribution of lipid changes in host cells may not be ruled out.

In order to investigate which biological activities mediate the inhibition of lung colonisation of F10-SR cells in animals fed a fish oil diet and injected with F10-SR cells, we examined immunohistochemically PCNA, bax/bcl-2 and VEGF/vWF, as markers of growth, apoptosis and vascularisation, respectively. This investigation revealed that the reduced lung colonisation of melanoma cells in animals fed a fish oil diet is associated with an increased apoptotic activity, as shown by the enhancement of immunoreactivity of bax combined with a visualisation of nuclear DNA fragments in pulmonary colonies. This finding is in agreement with the positive correlation between the consumption of fish oil diets and apoptotic activity reported in several studies $^{(40-42)}$. The unchanged PCNA immunoreactivity in the lung colonies developed in animals fed a fish oil diet suggests that the antimetastatic effect of dietary n-3 PUFA is not mediated by an inhibition of tumour cell proliferation, a finding in contrast with the selective antiproliferative effects of $n-3$ PUFA observed in different systems of tumour cells grown in tissue culture ${ }^{(43-47)}$. However, the artificial conditions produced in in vitro studies, not comparable with the complex in vivo tumour microenvironment, limit the significance of the data derived from in vitro studies regarding the influence of specific lipid components, including PUFA, on tumour growth (for a review, see Diggle ${ }^{(48)}$ ).

The diminution of microvessel density found in pulmonary colonies developed from F10-SR cells in animals fed a fish oil diet might contribute to the antimetastatic effect of dietary $n-3$ PUFA. This observation is supported by the finding that the reduction of murine mammary tumour growth by dietary $n-3$ PUFA is mediated by an inhibition of angiogenesis ${ }^{(49,50)}$. The association of a reduced microvessel density with a high VEGF immunoreactivity in pulmonary colonies developed in animals fed a fish oil diet is quite an unexpected 

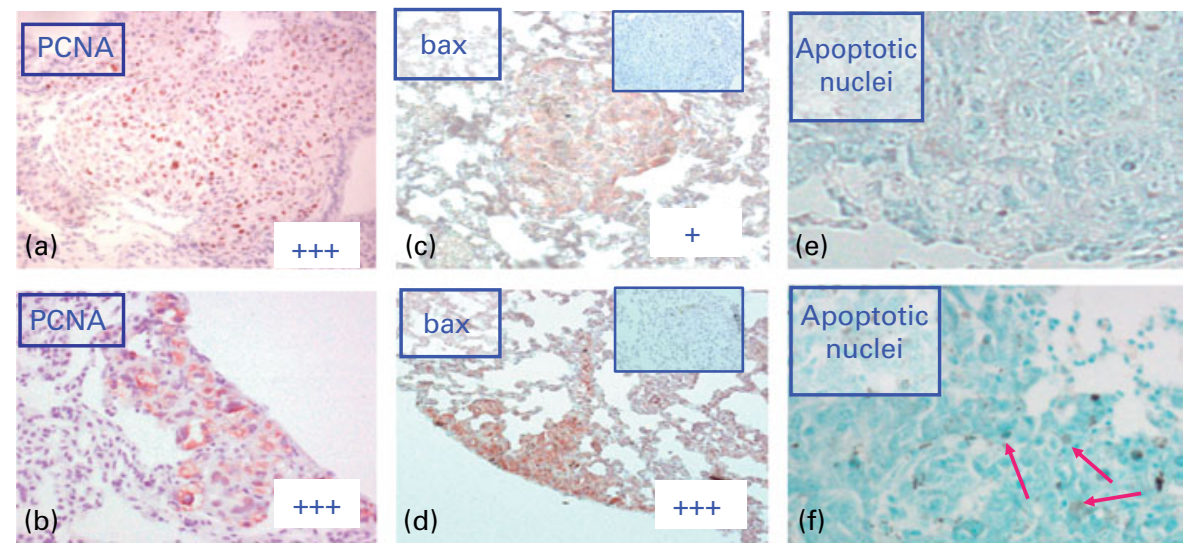

Fig. 2. Immunoreactivities of proliferating cell nuclear antigen (PCNA), bax and bcl-2 (see inserts in c and d) and visualisation of apoptotic nuclei ( $\leftarrow$ ) in pulmonary colonies developed from F10-SR cells in animals fed maize oil (a, c, e) or fish oil (b, d, f) diets. The immunoreactivity of each marker was evaluated as reported in Materials and methods. + , Focal staining (less than $10 \%) ;+++$, strong staining $(>50 \%$ stained cells).

finding. However, it is possible that $n-3$ PUFA inhibit vascularisation by counteracting the stimulatory activity of VEGF on vascular endothelia. Indeed, the competition between dietary $n$-3 PUFA and $n$ - 6 PUFA inhibits the synthesis of PGE2 ${ }^{(51,52)}$, an essential factor in angiogenesis ${ }^{(53,54)}$. Moreover, Salcedo et al. ${ }^{(54)}$ showed that the inhibition of PGE2 synthesis abrogates the VEGF-mediated expression of CXCR4, a key receptor in angiogenesis.

In conclusion, the data derived from the present study point out the possibility that even limited amounts of $n-3$ PUFA in the diet are able to inhibit the reproduction of experimental metastases from F10-SR cells. The reduced formation of metastases from F10-SR cells transplanted into animals fed an $n$-3 PUFA-rich diet might be the final step of a series of phenomena, consisting of: a decreased generation of PGE2 - reduced microvessel density - hypoxia - induction of apoptosis. This sequence, however, does not rule out the possibility that promotion of apoptosis and inhibition of angiogenesis represent two independent responses to the accumulation of $n-3$ PUFA.
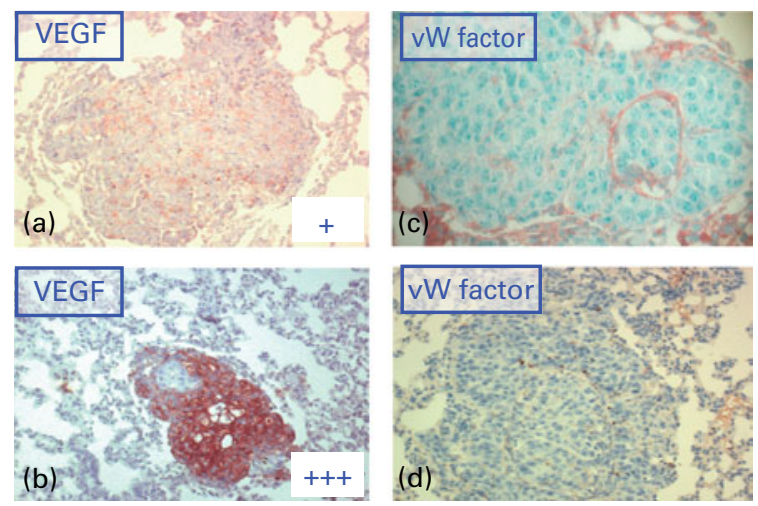

Fig. 3. Immunoreactivities of vascular endothelial growth factor (VEGF) and endothelial von Willebrand factor (VWF) in pulmonary colonies developed from F10-SR cells in animals fed maize oil $(a, c)$ or fish oil $(b, d)$ diets. The immunoreactivity of each marker was evaluated as reported in Materials and methods. + , Focal staining (less than $10 \%) ;+++$, strong staining $(>50 \%$ stained cells). The mean number of vWF-positive cells/microscopic field was ( $n$ 10) 41.6 (SEM 3.6) in (c) and 22.0 (SEM 1.9) in (d) $(P<0.05)$.

\section{Acknowledgements}

The present study was supported by Ente Fondazione Cassa di Risparmio di Firenze (Italy) and by Ente Fondazione Cassa di Risparmio di Lucca (Italy). None of the authors has conflicts of interest with respect to the study. S. R. and A. M. designed the study and prepared the paper. A. M. and N. K. contributed to the successful execution of experimental work. L. C. and G. M. contributed to the lipid analysis.

\section{References}

1. Byers $\mathrm{T} \&$ \& Graham S (1984) The epidemiology of diet and cancer. Adv Cancer Res 41, 1-69.

2. Willett WC \& MacMahon B (1984) Diet and cancer - an overview. $N$ Engl J Med 310, 633-638.

3. Carroll KK \& Khor HT (1975) Dietary fat in relation to tumorigenesis. Prog Biochem Pharmacol 10, 308-353.

4. Hubbard NE \& Erickson KL (1989) Effect of dietary linoleic acid level on lodgement, proliferation and survival of mammary tumor metastases. Cancer Lett 44, 117-125.

5. Rose DP, Connolly JM \& Meschter CL (1991) Effect of dietary fat on human breast cancer growth and lung metastasis in nude mice. J Natl Cancer Inst 83, 1491-1495.

6. Katz EB \& Boylan ES (1989) Effect of the quality of dietary fat on tumor growth and metastasis from a rat mammary adenocarcinoma. Nutr Cancer 12, 343-350.

7. Mannini A, Calzolari A, Calorini L, Mugnai G \& Ruggieri S (2006) The inhibition of lung colonization of B16-F10 melanoma cells in EFA-deficient animals is related to enhanced apoptosis and reduced angiogenesis. Clin Exp Metastasis 23, $159-165$

8. Hubbard NE, Chapkin RS \& Erickson KL (1988) Inhibition of growth and linoleate-enhanced metastasis of a transplantable mouse mammary tumor by indomethacin. Cancer Lett 43, $111-120$

9. Marks F, Muller-Decker K \& Furstenberger G (2000) A causal relationship between unscheduled eicosanoid signaling and tumor development: cancer chemoprevention by inhibitors of arachidonic acid metabolism. Toxicology 153, 11-26.

10. Sasaki S, Horacsek M \& Kesteloot H (1993) An ecological study of the relationship between dietary fat intake and breast cancer mortality. Prev Med 22, 187-202.

11. Caygill CP, Charlett A \& Hill MJ (1996) Fat, fish, fish oil and cancer. Br J Cancer 74, 159-164. 
12. Leitzmann MF, Stampfer MJ, Michaud DS, Augustsson K, Colditz GC, Willett WC \& Giovannucci EL (2004) Dietary intake of $n-3$ and $n-6$ fatty acids and the risk of prostate cancer. Am J Clin Nutr 80, 204-216.

13. Terry PD, Rohan TE \& Wolk A (2003) Intakes of fish and marine fatty acids and the risks of cancers of the breast and prostate and of other hormone-related cancers: a review of the epidemiologic evidence. Am J Clin Nutr 77, 532-543.

14. Lands WE, Hamazaki T, Yamazaki K, Okuyama H, Sakai K, Goto Y \& Hubbard VS (1990) Changing dietary patterns. Am J Clin Nutr 51, 991-993.

15. Kamano K, Okuyama H, Konishi R \& Nagasawa H (1989) Effects of a high-linoleate and a high- $\alpha$-linolenate diet on spontaneous mammary tumourigenesis in mice. Anticancer Res $\mathbf{9}$, 1903-1908.

16. Carroll KK \& Hopkins GJ (1979) Dietary polyunsaturated fat versus saturated fat in relation to mammary carcinogenesis. Lipids 14, 155-158.

17. Cave WT (1991) Dietary $n-3$ ( $\omega 3$ ) polyunsaturated fatty acid effects on animal tumorigenesis. FASEB $J$ 5, 2160-2166.

18. Karmali RA (1989) n-3 Fatty acids and cancer. J Intern Med Suppl 731, 197-200.

19. Jurkowski JJ \& Cave WT Jr (1985) Dietary effects of menhaden oil on the growth and membrane lipid composition of rat mammary tumors. J Natl Cancer Inst 74, 1145-1150.

20. Gabor H \& Abraham S (1986) Effect of dietary menhaden oil on tumor cell loss and the accumulation of mass of a transplantable mammary adenocarcinoma in BALB/c mice. J Natl Cancer Inst 76, $1223-1229$.

21. Reddy BS \& Maruyama H (1986) Effect of dietary fish oil on azoxymethane-induced colon carcinogenesis in male F344 rats. Cancer Res 46, 3367-3370.

22. Calviello G, Palozza P, Piccioni E, Maggiano N, Frattucci A, Franceschelli P \& Bartoli GM (1998) Dietary supplementation with eicosapentaenoic and docosahexaenoic acid inhibits growth of Morris hepatocarcinoma 3924A in rats: effects on proliferation and apoptosis. Int J Cancer 75, 699-705.

23. de Bravo MG, de Antueno RJ, Toledo J, De Tomás ME, Mercuri OF \& Quintans C (1991) Effects of an eicosapentaenoic and docosahexaenoic acid concentrate on a human lung carcinoma grown in nude mice. Lipids 26, 866-870.

24. Reich R, Royce L \& Martin GR (1989) Eicosapentaenoic acid reduces the invasive and metastatic activities of malig nant tumor cells. Biochem Biophys Res Commun 160, $559-564$

25. Iigo $\mathrm{M}$, Nakagawa $\mathrm{T}$, Ishikawa $\mathrm{C}$, Iwahori $\mathrm{Y}$, Asamoto $\mathrm{M}$, Yazawa K, Araki E \& Tsuda H (1997) Inhibitory effects of docosahexaenoic acid on colon carcinoma 26 metastasis to the lung. Br J Cancer 75, 650-655.

26. Rose DP \& Connolly JM (2000) Regulation of tumor angiogenesis by dietary fatty acids and eicosanoids. Nutr Cancer 37, 119-127.

27. Yam D, Peled A, Huszar M \& Shinitzky M (1997) Dietary fish oil suppresses tumor growth and metastasis of Lewis lung carcinoma in mice. J Nutr Biochem 8, 619-622.

28. Salem ML, Kishihara K, Abe K, Matsuzaki G \& Nomoto K (2000) n-3 Polyunsaturated fatty acids accentuate B16 melanoma growth and metastasis through suppression of tumoricida function of $\mathrm{T}$ cells and macrophages. Anticancer Res 20, $3195-3203$

29. Griffini P, Fehres O, Klieverik L, Vogels IM, Tigchelaar W, Smorenburg SM \& Van Noorden CJ (1998) Dietary omega-3 polyunsaturated fatty acids promote colon carcinoma metastasis in rat liver. Cancer Res 58, 3312-3319.

30. Cifone MA (1982) In vitro growth characteristics associated with benign and metastatic variants of tumor cells. Cancer Metastasis Rev 1, 335-347.
31. Larsson SC, Kumlin M, Ingelman-Sundberg M \& Wolk A (2004) Dietary long-chain $n-3$ fatty acids for the prevention of cancer: a review of potential mechanisms. Am J Clin Nutr 79, 935-945.

32. Malaguarnera L (2004) Implications of apoptosis regulators in tumorigenesis. Cancer Metastasis Rev 23, 367-387.

33. Li CY, Shan S, Cao Y \& Dewhirst MW (2000) Role of incipient angiogenesis in cancer metastasis. Cancer Metastasis Rev 19, $7-11$.

34. Hall PA, Levison DA, Woods AL, et al. (1990) Proliferating cell nuclear antigen (PCNA) immunolocalization in paraffin sections: an index of cell proliferation with evidence of deregulated expression in some neoplasms. J Pathol 162, 285-294.

35. Granville DJ, Carthy CM, Hunt DW \& McManus BM (1998) Apoptosis: molecular aspects of cell death and disease. Lab Invest 78, 893-913.

36. Ferrara N \& Gerber HP (2002) The role of vascular endothelial growth factor in angiogenesis. Acta Haematol 106, 148-156.

37. Müller AM, Hermanns MI, Skrzynski C, Nesslinger M, Müller KM \& Kirkpatrick CJ (2002) Expression of the endothelial markers PECAM-1, vWf, and CD34 in vivo and in vitro. Exp Mol Pathol 72, 221-229.

38. Folch J, Lees M \& Sloane Stanley GH (1957) A simple method for the isolation and purification of total lipides from animal tissues. J Biol Chem 226, 497-509.

39. Gavrieli Y, Sherman Y \& Ben-Sasson SA (1992) Identification of programmed cell death in situ via specific labeling of nuclear DNA fragmentation. J Cell Biol 119, 493-501.

40. Rose DP \& Connolly JM (1999) Omega-3 fatty acids as cancer chemopreventive agents. Pharmacol Ther 83, 217-244.

41. Chang WL, Chapkin RS \& Lupton JR (1998) Fish oil blocks azoxymethane-induced rat colon tumorigenesis by increasing cell differentiation and apoptosis rather than decreasing cell proliferation. J Nutr 128, 491-497.

42. Latham P, Lund EK \& Johnson IT (1999) Dietary n-3 PUFA increases the apoptotic response to 1,2-dimethylhydrazine, reduces mitosis and suppresses the induction of carcinogenesis in the rat colon. Carcinogenesis 20, 645-650

43. Mund RC, Pizato N, Bonatto S, Nunes EA, Vicenzi T, Tanhoffer R, de Oliveira HH, Curi R, Calder PC \& Fernandes LC (2007) Decreased tumor growth in Walker 256 tumor-bearing rats chronically supplemented with fish oil involves COX-2 and PGE2 reduction associated with apoptosis and increased peroxidation. Prostaglandins Leukot Essent Fatty Acids 76, 113-120.

44. Calviello G, Di Nicuolo F, Gragnoli S, Piccioni E, Serini S, Maggiano N, Tringali G, Navarra P, Ranelletti FO \& Palozza P (2004) $n$-3 PUFAs reduce VEGF expression in human colon cancer cells modulating the COX-2/PGE2 induced ERK-1 and -2 and HIF-1 $\alpha$ induction pathway. Carcinogenesis 25, 2303-2310

45. Narayanan BA, Narayanan NK, Desai D, Pittman B \& Reddy BS (2004) Effects of a combination of docosahexaenoic acid and 1,4-phenylene bis(methylene) selenocyanate on cyclooxygenase 2 , inducible nitric oxide synthase and $\beta$-catenin pathways in colon cancer cells. Carcinogenesis 25, 2443-2449.

46. Trombetta A, Maggiora M, Martinasso G, Cotogni P, Canuto RA \& Muzio G (2007) Arachidonic and docosahexaenoic acids reduce the growth of A549 human lung-tumor cells increasing lipid peroxidation and PPARs. Chem Biol Interact 165, 239-250.

47. Bégin ME, Ells G, Das UN \& Horrobin DF (1986) Differential killing of human carcinoma cells supplemented with $n-3$ and $n-6$ polyunsaturated fatty acids. J Natl Cancer Inst 77, 1053-1062.

48. Diggle CP (2002) In vitro studies on the relationship between polyunsaturated fatty acids and cancer: tumour or tissue specific effects? Prog Lipid Res 41, 240-253. 
49. Rose DP \& Connolly JM (1999) Antiangiogenicity of docosahexaenoic acid and its role in the suppression of breast cancer cell growth in nude mice. Int J Oncol 15, 1011-1015.

50. Mukutmoni-Norris M, Hubbard NE \& Erickson KL (2000) Modulation of murine mammary tumor vasculature by dietary n-3 fatty acids in fish oil. Cancer Lett 150, 101-109.

51. Horia E \& Watkins BA (2007) Complementary actions of docosahexaenoic acid and genistein on COX-2, PGE2 and invasiveness in MDA-MB-231 breast cancer cells. Carcinogenesis 28, 809-815.
52. Henderson CD, Black HS \& Wolf JE Jr (1989) Influence of omega- 3 and omega- 6 fatty acid sources on prostaglandin levels in mice. Lipids 24, 502-505.

53. Ziche M, Jones J \& Gullino PM (1982) Role of prostaglandin E1 and copper in angiogenesis. J Natl Cancer Inst 69, 475-482.

54. Salcedo R, Zhang X, Young HA, Michael N, Wasserman K, Ma WH, Martins-Green M, Murphy WJ \& Oppenheim JJ (2003) Angiogenic effects of prostaglandin E2 are mediated by upregulation of CXCR4 on human microvascular endothelial cells. Blood 102, 1966-1977. 